\title{
Bounded directional distance function models
}

\author{
Jesus T. Pastor ${ }^{1} \cdot$ Juan Aparicio $^{1}$ (D) - Javier Alcaraz ${ }^{1} \cdot$ Fernando Vidal $^{2}$. \\ Diego Pastor ${ }^{3}$
}

(c) Springer-Verlag GmbH Germany, part of Springer Nature 2018

\begin{abstract}
Bounded additive models in data envelopment analysis (DEA) under the assumption of constant returns to scale (CRS) were recently introduced in the literature (Cooper et al. in J Product Anal 35(2):85-94, 2011; Pastor et al. in J Product Anal 40:285-292, 2013; Pastor et al. in Omega 56:16-24, 2015). In this paper, we propose to extend the so far generated knowledge about bounded additive models to the family of directional distance function (DDF) models in DEA, giving rise to a completely new subfamily of bounded or partially-bounded CRS-DDF models. We finally check the new approach on a real agricultural panel data set estimating efficiency and productivity change over time, resorting to the Luenberger indicator in a context where at least one variable is naturally bounded.
\end{abstract}

Keywords Data envelopment analysis - Directional distance functions · Bounded or partially-bounded DEA CRS-models

\section{Introduction}

Data envelopment analysis (DEA) is a non-parametric methodology based on Mathematical Programming for the assessment of relative efficiency of a set of homogeneous decision making units (DMUs) that use different amounts of the same inputs to produce different amounts of the same outputs. DEA models generally provide efficiency-or inefficiency-measures for each of the assessed DMUs as well as information on the targets that have been used in the efficiency assessment in the case of inefficient DMUs.

$凶$ Juan Aparicio

j.aparicio@umh.es

1 Center of Operations Research (CIO), University Miguel Hernandez of Elche (UMH), 03202 Elche, Alicante, Spain

2 Environmental Economics Department, University Miguel Hernandez of Elche (UMH), 03212 Orihuela (Alicante), Spain

3 Physical and Sports Education, University Miguel Hernandez of Elche (UMH), 03202 Elche, Alicante, Spain 
In recent times, DEA has grown into a powerful quantitative tool for measuring and evaluating performance. In fact, this technique has been successfully applied to a multitude of different entities engaged in a wide variety of activities: banking, health care, education, sports, agriculture, manufacturing and so on (see Liu et al. 2013; Toloo et al. 2017, 2018).

Bounded additive models have been introduced quite recently in the DEA literature (Cooper et al. 2011). The justification for defining bounded measures follows. The usual benchmarks yielded by traditional models in DEA may exhibit properties that are undesirable from a managerial and practical point of view. When constant returns to scale (CRS) is assumed in the evaluation of technical efficiency, a target in an input-oriented model can be a point that requires the usage of less input than in any of the given observations. Similarly, in output-oriented models, the target point may require the production of more output than what is currently observed in the data set. Additionally, in a non-oriented model, a target may need one of these two requirements. Such input and output targets, which result from the extrapolation outside of the observed data set under the CRS assumption, may be inappropriate or undesirable in some situations. One potential problem is the lack of empirical evidence to support the feasibility of these points, e.g., when the sample matches up the whole population. Another potential problem may arise if there are natural upper bounds for the variable values, for instance in the case of ratio variables (see, e.g., Olesen et al. 2015), or percentages (e.g., unemployment rate as a percentage of the work force). Indeed, if we use ratio variables and/or variables expressed in terms of percentages, it may also be desirable to specifically only allow CRS when the nature of these variables implies that the size effect of the units being assessed has been removed. In this way, bounded additive models were introduced in order to deal with naturally lower and upper bounded inputs and outputs, while working within a CRS context at the same time.

Since their inception, the bounded additive models have been applied to several sectors and situations. Chronologically speaking, Vidal et al. (2013) estimated productivity change over time in the Spanish wine sector. Vidal et al. (2014) applied these models to measure the evolution of technical efficiency of a set of Spanish virgin olive oil Designations of Origin. Pastor et al. (2014) studied the effectiveness of tourism websites in Mediterranean countries by applying bounded models. More recently, Rashidi and Saen (2015) have developed and checked a bounded additive measure based on green indicators in order to calculate eco-efficiency.

Bounded DEA models were introduced in Cooper et al. (2011), as we have already mentioned, and extended in Pastor et al. (2013, 2015). Their origin was a direct consequence of the introduction of a weighted additive model under Variable Returns to Scale (VRS) with a new structure of weights, which was named BAM (Bounded Adjusted Measure; Cooper et al. 2011). This model was designed in order to achieve a new efficiency measure, so as to improve the discriminatory power of the previous defined Range Adjusted Measure (RAM; Cooper et al. 1999). In the above first mentioned paper the extension of the BAM model assuming any returns to scale was proposed, and, particularly, the CRS-BAM model was formulated. Its formulation was similar to the BAM model working under VRS, with a basic difference, besides the usual deletion of the convexity constraint, input-lower range bound and 
output-upper range bound restrictions had to be added to the corresponding linear programming model. The new added constraints guaranteed that the projection of the point being rated belonged to an associated bounded production possibility set under CRS. This was the first bounded DEA model proposed in the specialized literature. Its formulation was refined by Pastor et al. (2013) adding a set of "point-slacks" to each projection which proved to be critical for obtaining the characterization of the Pareto-efficient points of the bounded technology. Very recently, Pastor et al. (2015) created the Enhanced BAM (EBAM) under CRS, introducing a new set of bounds, identified as "comprehensive-bounds", which have the property of maintaining the original CRS efficient projections as points of the corresponding bounded technology. Moreover, and when certain inputs and/or outputs have natural lower and/or upper bounds, these authors introduced the so called CRS partially-bounded weighted additive model, which also gave rise to two possible efficiency measures, the BAM under CRS and the EBAM under CRS for partially-bounded additive models. The first one adds to the non-bounded variables their corresponding range-bounds, while the second one considers comprehensive bounds instead.

All the aforementioned bounded models were introduced considering, at each point being rated, the maximization of a weighted $\mathrm{L}_{1}$-path towards the Pareto-efficient frontier. In this sense, what we propose in this paper is to extend the notion of bounded CRS weighted additive model to other types of well-known CRS-DEA models where the path towards the frontier is directional, that is, it follows the direction of a prespecified vector, and Pareto-efficiency is not required. Moreover, instead of generating an efficiency measure, the new model under consideration generates an inefficiency measure. Being more specific, we are going to focus our attention on the directional distance function (DDF) model, which is one of the most widely used DEA inefficiency models. Our purpose is to introduce the theoretical tools that will allow us to consider CRS-DDF models with at least one of its inputs-or outputs-lower-bounded-or upper bounded. One of the potential uses of the new DDF model is its application to the field of efficiency and/or productivity measurement over time when one or more of the variables are ratios or are expressed as percentages.

The productivity analysis offers two different ways of measuring productivity change over time, namely Malmquist indexes or Luenberger indicators. The latter were designed for dealing with DDFs, or, in a more open context, for dealing with additive inefficiency measures. In any case, the specialized literature for estimating productivity change over time recommends using constant returns to scale models (see Grifell-Tatjé and Lovell 1995; Ray and Desli 1997; Balk 2001; Lovell 2003; Kapelko et al. 2015). However, a priori this assumption cannot be combined with, for example, percentage variables, since in this case, the obtained projections could be greater than 100. In this respect, we introduce the necessary tools for mixing CRS and this type of inputs and outputs, defining the appropriate CRS-bounded DDF model and showing how it can be utilized for measuring productivity change through the corresponding Luenberger indicator.

The paper unfolds as follows. In Sect. 2 we revise the main definitions and results associated with bounded additive models. The bounded CRS-DDF model is introduced in Sect. 3, while Sect. 4 is devoted to introducing the partially-bounded CRS-DDF model. Section 5 considers the well-known Luenberger indicator for measuring pro- 
ductivity change based on the new partially bounded-or bounded-DDF models, showing its decomposition into efficiency change and technical change. Section 6 presents an illustrative empirical example and, finally, Sect. 7 concludes.

\section{A revision of the bounded additive models}

In this section, we revise the basic definitions and results linked to the bounded additive models (see Cooper et al. 2011; Pastor et al. 2013, 2015). They will be called upon later in the text.

The typical formulation of a DEA model that measures inefficiency corresponds to a linear program that evaluates the technical inefficiency of a fixed unit selected among a finite sample of units. The finite sample of units defines $T$, the production possibility set.

Let us consider the sample of $n$ units to be evaluated. Unit $j \in\{1,2, \ldots, n\}$ uses a specific amount of $m$ inputs, $\boldsymbol{x}_{j}=\left(x_{1 j}, \ldots, x_{m j}\right) \in R_{++}^{m}$, to produce a certain amount of $s$ outputs $\boldsymbol{y}_{j}=\left(y_{1 j}, \ldots, y_{s j}\right) \in R_{++}^{s}$. If we assume that the technology satisfies CRS, the definition of $T$ follows (see Charnes et al. 1978).

$T=\left\{(\boldsymbol{x}, \boldsymbol{y}) \in R_{+}^{m+s}: \sum_{j=1}^{n} \lambda_{j} x_{i j} \leq x_{i}, \quad \forall i, \quad \sum_{j=1}^{n} \lambda_{j} y_{r j} \geq y_{r}, \quad \forall r, \quad \lambda_{j} \geq 0, \quad \forall j\right\}$.

Let us introduce some additional notation. Let $\underline{x}_{i} \leq x_{i}, i=1, \ldots, m$, be a lower bound for each input $i$ and $\bar{y}_{r} \geq y_{r}, r=1, \ldots, s$, be an upper bound for each output $r$. Now, under CRS, we define the bounded technology, $T^{B}$, as follows:

$T^{B}=\left\{(\boldsymbol{x}, \boldsymbol{y}) \in R_{+}^{m+s}:(\boldsymbol{x},-\boldsymbol{y}) \geq \sum_{j=1}^{n} \lambda_{j}\left(\boldsymbol{x}_{j},-\boldsymbol{y}_{j}\right), \quad \lambda_{j} \geq 0, \quad \forall j ; \quad x_{i} \geq \underline{x}_{i}, \quad \forall i ; \quad y_{r} \leq \bar{y}_{r}, \quad \forall r\right\}$.

By definition, the bounded technology (2) is the result of adding lower bounds for inputs and upper bounds for outputs to the original (unbounded) technology (1). Let us observe that upper bounds for inputs or lower bounds for outputs are superfluous because the projection of an inefficient point towards the efficient frontier in any DEA model either reduces inputs or increases outputs or both.

Additionally, the weakly efficient frontier of $T^{B}$ is defined as

$$
\partial^{w}\left(T^{B}\right):=\left\{(\boldsymbol{x}, \boldsymbol{y}) \in T^{B}: \hat{\boldsymbol{x}}<\boldsymbol{x}, \hat{\boldsymbol{y}}>\boldsymbol{y} \Rightarrow(\hat{\boldsymbol{x}}, \hat{\boldsymbol{y}}) \notin T^{B}\right\} .
$$

Following Koopmans (1951), in order to measure technical efficiency in the Pareto sense, isolating a certain subset of $\partial^{w}\left(T^{B}\right)$ is necessary. We are referring to the strongly efficient frontier, defined as

$$
\partial^{s}\left(T^{B}\right):=\left\{(\boldsymbol{x}, \boldsymbol{y}) \in T^{B}: \hat{\boldsymbol{x}} \leq \boldsymbol{x}, \hat{\boldsymbol{y}} \geq \boldsymbol{y},(\hat{\boldsymbol{x}}, \hat{\boldsymbol{y}}) \neq(\boldsymbol{x}, \boldsymbol{y}) \Rightarrow(\hat{\boldsymbol{x}}, \hat{\boldsymbol{y}}) \notin T^{B}\right\} .
$$


In words, $\partial^{S}\left(T^{B}\right)$ is the set of all the Pareto-Koopmans efficient points of $T^{B}$.

The next proposition characterizes the set of points of the bounded technology $T^{B}$ (Pastor et al. 2013), resorting in this case to the set of Pareto-efficient DMUs, denoted as $E$.

Proposition 1 (Pastor et al. 2013) The bounded technology, $T^{B}$, can be equivalently rewritten as

$$
T^{B}=\left\{(\boldsymbol{x}, \boldsymbol{y}) \in R_{+}^{m} \times R_{+}^{s}:(\boldsymbol{x},-\boldsymbol{y}) \geq \sum_{j \in E} \lambda_{j}\left(\boldsymbol{x}_{j},-\boldsymbol{y}_{j}\right) ; \lambda_{j} \geq 0, \forall j ; \quad x_{i} \geq \underline{x}_{i}^{x}, \quad \forall i ; \quad y_{r} \leq \bar{y}_{r}, \quad \forall r\right\} .
$$

Let us now additionally introduce the formulation of input and output range-bounds. For any input $i$ its lower range-bound is defined as ${ }_{x_{i}}^{R} \min \left\{x_{i j}, j=1, \ldots, n\right\}$, while for each output $r$ its upper range-bound is defined as $\bar{y}_{r}^{R}=\max \left\{y_{r j}, j=1, \ldots, n\right\}$. These (side-) bounds are crucial for defining the CRS version of the Bounded Adjusted Measure of inefficiency. Its formulation follows (see Pastor et al. 2013, p. 288).

$$
\begin{aligned}
& B A M\left(\boldsymbol{x}_{k}, \boldsymbol{y}_{k}\right)=\operatorname{Max} \frac{1}{m+s}\left(\sum_{i=1}^{m} \frac{s_{i k}^{-}}{x_{i k}-\underline{x}_{i}^{R}}+\sum_{r=1}^{s} \frac{s_{r k}^{+}}{\overline{\bar{y}}_{r}^{R}-y_{r k}}\right) \\
& \text { s.t. } \\
& \sum_{j \in E} \lambda_{j} x_{i j}+\tau_{i k}^{-}=x_{i k}-s_{i k}^{-}, \\
& i=1, \ldots, m(6.2) \\
& \sum_{j \in E} \lambda_{j} y_{r j}-\tau_{r k}^{+}=y_{r k}+s_{r k}^{+} \quad r=1, \ldots, s \\
& \sum_{j \in E} \lambda_{j} x_{i j}+\tau_{i k}^{-} \geq \underline{x}_{i}^{R}, \quad i=1, \ldots, m(6.4) \\
& \sum_{j \in E} \lambda_{j} y_{r j}-\tau_{r k}^{+} \leq \bar{y}_{r}^{R}, \quad r=1, \ldots, s \text { (6.5) } \\
& \lambda_{j} \geq 0, \quad j \in E \\
& s_{i k}^{-} \geq 0, \\
& \text { j } \\
& s_{r k}^{+} \geq 0 \\
& i=1, \ldots, m \\
& r=1, \ldots, s \\
& \tau_{i k}^{-} \geq 0 \text {, } \\
& \tau_{r k}^{+} \geq 0 \text {, } \\
& i=1, \ldots, m(6.9) \\
& r=1, \ldots, s
\end{aligned}
$$

Model (6) can be simplified and is equivalent to the next model (7) thanks to (6.9) and (6.10) and the maximization criterion, deleting in this way the second set of pointslacks of (6), which in general are relevant for identifying the strongly efficient points of the bounded technology. In what follows the last mentioned identification plays a 
minor role, because when dealing with bounded DDFs the projections belong to the weakly efficient frontier and, in general, not to the strongly efficient frontier.

$$
\begin{aligned}
& B A M\left(\boldsymbol{x}_{k}, \boldsymbol{y}_{k}\right)=\operatorname{Max} \frac{1}{m+s}\left(\sum_{i=1}^{m} \frac{s_{i k}^{-}}{x_{i k}-\underline{x}_{i}^{R}}+\sum_{r=1}^{s} \frac{s_{r k}^{+}}{\bar{y}_{r}^{R}-y_{r k}}\right) \\
& \text { s.t. } \\
& \sum_{j \in E} \lambda_{j} x_{i j} \leq x_{i k}-s_{i k}^{-} \\
& i=1, \ldots, m(7.2) \\
& \sum_{j \in E} \lambda_{j} y_{r j} \geq y_{r k}+s_{r k}^{+} \\
& r=1, \ldots, s \\
& x_{i k}-s_{i k}^{-} \geq \underline{x}^{R} \text {, } \\
& y_{r k}+s_{r k}^{+} \leq \bar{y}_{r}^{i} \text {, } \\
& \lambda_{j} \geq 0 \text {, } \\
& s_{i k}^{-} \geq 0 \text {, } \\
& s_{r k}^{+} \geq 0 \text {, } \\
& i=1, \ldots, m(7.4) \\
& r=1, \ldots, s \\
& j \in E \\
& i=1, \ldots, m(7.7) \\
& r=1, \ldots, s \quad(7.8)
\end{aligned}
$$

Constraints (7.4) and (7.5) guarantee that the projection of point $\left(\boldsymbol{x}_{k}, \boldsymbol{y}_{k}\right)$, as given by $\left(x_{k}-s_{k}^{-*}, y_{k}+s_{k}^{+*}\right)$, belongs to the bounded production possibility set $T^{B}$ with $\underline{x}_{i}=\underline{x}_{i}^{R}, \quad \forall i$ and $\bar{y}_{r}=\bar{y}_{r}^{R}, \quad \forall r$. Indeed, it can be proved that these targets belong to the strongly efficient frontier of $T^{B}$ (see Pastor et al. 2013), as the next proposition states.

Proposition 2 (Pastor et al. 2013) Let $\left(\lambda^{*}, \boldsymbol{s}^{-*}, \boldsymbol{s}^{+*}\right)$ be an optimal solution of (7). Then, $\left(\boldsymbol{x}_{k}-\boldsymbol{s}_{k}^{-*}, \boldsymbol{y}_{k}+\boldsymbol{s}_{k}^{+*}\right) \in \partial^{s}\left(T^{B}\right)$.

Regarding the properties satisfied by $B A M$, we show the following result.

Proposition 3 (Cooper et al. 2011) The Bounded Adjusted Measure of inefficiency meets the following properties.

(a) $0 \leq B A M\left(\boldsymbol{x}_{k}, \boldsymbol{y}_{k}\right) \leq 1$.

(b) $B A M\left(\boldsymbol{x}_{k}, \boldsymbol{y}_{k}\right)=0$ if and only if $\left(\boldsymbol{x}_{k}, \boldsymbol{y}_{k}\right) \in \partial^{s}\left(T^{B}\right)$.

(c) $B A M\left(\boldsymbol{x}_{k}, \boldsymbol{y}_{k}\right)$ is weakly monotonic.

(d) $B A M\left(\boldsymbol{x}_{k}, \boldsymbol{y}_{k}\right)$ is units invariant.

\section{The CRS bounded DDF model}

In this section, we extend the notion of bounded models, first introduced in connection with weighted additive models, to directional distance function models in DEA, defining the Bounded CRS-DDF model. Additionally, we prove some interesting properties for this new inefficiency measure.

First of all, let us formulate the definition of the directional distance function, given a general technology $T$ and a reference vector $\boldsymbol{g}=\left(-\boldsymbol{g}^{I}, \boldsymbol{g}^{O}\right) \neq \mathbf{0}_{m+s}, \boldsymbol{g}^{I} \in R_{+}^{m}$, 
$\boldsymbol{g}^{O} \in R_{+}^{s}$ when the rated point $(\boldsymbol{x}, \boldsymbol{y})$ belongs to $T$ (Chambers et al. 1996a, 1998), as follows. ${ }^{1}$

$$
D D F\left(\boldsymbol{x}, \boldsymbol{y} ; \boldsymbol{g}^{I}, \boldsymbol{g}^{O}\right)=\max \left\{\beta:\left(\boldsymbol{x}-\beta \boldsymbol{g}^{I}, \boldsymbol{y}+\beta \boldsymbol{g}^{O}\right) \in T\right\}
$$

Any DDF is identified by specifying a directional vector $\boldsymbol{g}=\left(-\boldsymbol{g}^{I}, \boldsymbol{g}^{O}\right)$ at each point being rated. In order to measure the inefficiency associated to a specific unit of the finite sample, the DDF projects the unit onto the weakly efficient frontier of the technology (not necessarily the strongly part of the efficient frontier) along the positive semi-ray defined by vector $\boldsymbol{g}$. Additionally, $\boldsymbol{g}$ may be constant, i.e., $\boldsymbol{g}$ is the same vector for all the units being rated, or $\boldsymbol{g}$ may be variable, i.e., it is a specific vector for each unit. In the latter case and for unit $\left(\boldsymbol{x}_{k}, \boldsymbol{y}_{k}\right)$, we write $\boldsymbol{g}_{k}$ instead of $\boldsymbol{g}$. As said before, the projection of unit $\left(\boldsymbol{x}_{k}, \boldsymbol{y}_{k}\right)$ onto the weakly efficient frontier is the intersection of the semi-ray $\left\{\left(\boldsymbol{x}_{k}, \boldsymbol{y}_{k}\right)+\beta_{k}\left(-\boldsymbol{g}_{k}^{I}, \boldsymbol{g}_{k}^{O}\right), \quad \beta_{k} \geq 0\right\}$ with the weakly efficient frontier. The specific value of scalar $\beta_{k}$ that determines this point of intersection is interpreted as the technical inefficiency of point $\left(\boldsymbol{x}_{k}, \boldsymbol{y}_{k}\right)$ measured by the DDF. Recent details on the DDF can be found in Briec and Kerstens (2009a, b).

By analogy with (8), the Bounded Directional Distance Function (BDDF) model may be defined simply by substituting $T$ by $T^{B}$ :

$$
B D D F\left(\boldsymbol{x}, \boldsymbol{y} ; \boldsymbol{g}^{I}, \boldsymbol{g}^{O}\right)=\max \left\{\beta:\left(\boldsymbol{x}-\beta \boldsymbol{g}^{I}, \boldsymbol{y}+\beta \boldsymbol{g}^{O}\right) \in T^{B}\right\} .
$$

Its formulation as a linear programming model under CRS follows from Proposition 1 and is similar to model (7). ${ }^{2}$

$$
\begin{aligned}
& \operatorname{BDDF}_{C R S}\left(\boldsymbol{x}_{k}, \boldsymbol{y}_{k} ; \boldsymbol{g}_{k}^{I}, \boldsymbol{g}_{k}^{O}\right)=\underset{\text { s.t. }}{\operatorname{Max}} \beta_{k} \\
& \sum_{j \in E} \lambda_{j k} x_{i j} \leq x_{i k}-\beta_{k} g_{k i}^{I}, \quad i=1, \ldots, m(10.2) \\
& \sum_{j \in E} \lambda_{j k} y_{r j} \geq y_{r k}+\beta_{k} g_{k r}^{O}, \quad r=1, \ldots, s \\
& x_{i k}-\beta_{k} g_{k i}^{I} \geq \underline{x}_{i}, \quad i=1, \ldots, m(10.4) \\
& \begin{array}{ll}
y_{r k}+\beta_{k} g_{k r}^{O} \leq \bar{y}_{r}^{i}, & r=1, \ldots, s \\
\lambda_{j k} \geq 0, & j \in E
\end{array}
\end{aligned}
$$

\footnotetext{
${ }^{1}$ Luenberger (1992) introduced the concept of benefit function as a representation of the amount that an individual is willing to trade, in terms of a specific reference commodity bundle $\mathbf{g}$, for the opportunity to move from a consumption bundle to a utility threshold. Luenberger also defined a so-called shortage function (1992, p. 242, Definition 4.1), which basically measures the amount by which a specific plan is short of reaching the frontier of the technology. Later, Chambers et al. (1996a, 1998) redefined the benefit function and the shortage function as the directional distance function.

${ }^{2}$ Since we are interesting in measuring productivity, we will assume from now on CRS. The definition of a Bounded DDF model under any other returns to scale assumption is straightforward.
} 
The optimal value of (10), $\beta_{k}^{*}$, is directly related to the "distance" from the rated DMU to the weakly efficient frontier of the bounded technology $T^{B}$ measured along the semi-ray defined through the corresponding directional vector.

The next proposition relates efficiency with the optimal value of (10).

Proposition 4 If $B D D F_{C R S}\left(\boldsymbol{x}_{k}, \boldsymbol{y}_{k} ; \boldsymbol{g}_{k}^{I}, \boldsymbol{g}_{k}^{O}\right)=0$, then $\left(\boldsymbol{x}_{k}, \boldsymbol{y}_{k}\right) \in \partial^{w}\left(T^{B}\right)$.

Proof Let us assume that $B D D F_{C R S}\left(\boldsymbol{x}_{k}, \boldsymbol{y}_{k} ; \boldsymbol{g}_{k}^{I}, \boldsymbol{g}_{k}^{O}\right)=0$. If $\left(\boldsymbol{x}_{k}, \boldsymbol{y}_{k}\right) \notin \partial^{w}\left(T^{B}\right)$, then by expression (3) there exists a point $(\hat{\boldsymbol{x}}, \hat{\boldsymbol{y}})$ that belongs to $T^{B}$ such that $\hat{x}_{i}<$ $x_{i k}, \forall i$, and $\hat{y}_{r}>y_{r k}, \forall r$. Then by Proposition 1 we have that $\exists \hat{\lambda} \geq 0$ so that $\hat{x}_{i} \geq \sum_{j \in E} \hat{\lambda}_{j} x_{i j}, \forall i, \hat{y}_{r} \leq \sum_{j \in E} \hat{\lambda}_{j} y_{r j}, \forall r, \hat{x}_{i} \geq \underline{x}_{i}, \forall i$ and $\hat{y}_{r} \leq \bar{y}_{r}, \forall r$. As a consequence, $(\hat{\beta}, \hat{\lambda})$ with $\hat{\beta}:=\min _{i=1, \ldots, m,}\left\{\frac{x_{i k}-\hat{x}_{i}}{g_{i k}^{I}}, \frac{\hat{y}_{r}-y_{r k}}{g_{r k}^{O}}\right\}$, is a feasible solution

$$
\begin{array}{r}
r=1, \ldots, s \\
\text { such that } \\
g_{i k}^{I}, g_{r k}^{O} \neq 0
\end{array}
$$

of model (10). Note, however, that $\hat{\beta}>0$ since $\hat{x}_{i}<x_{i k}, \forall i$ and $\hat{y}_{r}>y_{r k}, \forall r$. This is a contradiction because $B D D F_{C R S}\left(\boldsymbol{x}_{k}, \boldsymbol{y}_{k} ; \boldsymbol{g}_{k}^{I}, \boldsymbol{g}_{k}^{O}\right)=0$. Therefore, point $(\hat{\boldsymbol{x}}, \hat{\boldsymbol{y}})$ cannot belong to $T^{B}$ and, applying expression (3), we conclude that $\left(\boldsymbol{x}_{k}, \boldsymbol{y}_{k}\right) \in$ $\partial^{w}\left(T^{B}\right)$.

The remaining properties satisfied by the Bounded CRS Directional Distance Function, however, depend on the characteristics of the selected directional vector. In this respect, we will go on to show two interesting properties, which correspond to properties (c) and (d) of Proposition 3.

Proposition 5 Let $\left(\boldsymbol{x}_{k}, \boldsymbol{y}_{k}\right) \in T^{B}, \boldsymbol{g}_{k}=\left(-\boldsymbol{g}_{k}^{I}, \boldsymbol{g}_{k}^{O}\right) \neq \mathbf{0}_{m+s}, \boldsymbol{g}_{k}^{I} \in R_{+}^{m}, \boldsymbol{g}_{k}^{O} \in R_{+}^{s}$ or, alternatively, $\boldsymbol{g}=\left(-\boldsymbol{g}^{I}, \boldsymbol{g}^{O}\right) \neq 0_{m+s}, \boldsymbol{g}^{I} \in R_{+}^{m}, \boldsymbol{g}^{O} \in R_{+}^{S}$. Then the Bounded CRS Directional Distance Function defined by (10) meets the following properties.

(a) Let $\boldsymbol{t}^{-}=\left(t_{1}^{-}, \ldots, t_{m}^{-}\right) \in R_{+}^{m}$ and $\boldsymbol{t}^{+}=\left(t_{1}^{+}, \ldots, t_{s}^{+}\right) \in R_{+}^{s}$ such that $\left(\boldsymbol{x}_{k}+\boldsymbol{t}^{-}, \boldsymbol{y}_{k}-\boldsymbol{t}^{+}\right) \in T^{B}$. Then

$B D D F_{C R S}\left(\boldsymbol{x}_{k}, \boldsymbol{y}_{k} ; \boldsymbol{g}^{I}, \boldsymbol{g}^{O}\right) \leq B D D F_{C R S}\left(\boldsymbol{x}_{k}+\boldsymbol{t}^{-}, \boldsymbol{y}_{k}-\boldsymbol{t}^{+} ; \boldsymbol{g}^{I}, \boldsymbol{g}^{O}\right)$.

(b) If the components of $\boldsymbol{g}_{k}$ and $\boldsymbol{g}$ are expressed in the units of measurement of the observed data, then $B D D F_{C R S}\left(\boldsymbol{x}_{k}, \boldsymbol{y}_{k} ; \boldsymbol{g}_{k}^{I}, \boldsymbol{g}_{k}^{O}\right)$ and $B D D F_{C R S}\left(\boldsymbol{x}_{k}, \boldsymbol{y}_{k} ; \boldsymbol{g}^{I}, \boldsymbol{g}^{O}\right)$ are units invariant.

Proof (a) Let $\left(\beta_{k}^{*}, \lambda_{k}^{*}\right)$ be a feasible solution of model (10) when $\left(\boldsymbol{x}_{k}, \boldsymbol{y}_{k}\right)$ is assessed. We are going to prove that $\left(\beta_{k}^{*}, \lambda_{k}^{*}\right)$ is a feasible solution of model (10) when $\left(\boldsymbol{x}_{k}+\boldsymbol{t}^{-}, \boldsymbol{y}_{k}-\boldsymbol{t}^{+}\right)$is evaluated. Regarding constraint (10.2), $\sum_{j \in E} \lambda_{j k}^{*} x_{i j} \leq$ $x_{i k}-\beta_{k}^{*} g_{i}^{I} \leq x_{i k}+t_{i}^{-}-\beta_{k}^{*} g_{i}^{I}, \quad \forall i$, and regarding constraint (10.4), $x_{i k}+t_{i}^{-}-$ $\beta_{k}^{*} g_{i}^{I} \geq x_{i k}-\beta_{k}^{*} g_{i}^{I} \geq \underline{x}_{i}, \quad \forall i$. A similar statement can be proved regarding the two output constraints, (10.3) and (10.5). In this way, $\left(\beta_{k}^{*}, \lambda_{k}^{*}\right)$ is a feasible solution of model (10) when $\left(\boldsymbol{x}_{k}+\boldsymbol{t}^{-}, \boldsymbol{y}_{k}-\boldsymbol{t}^{+}\right)$is evaluated and, consequently, $B D D F_{C R S}\left(\boldsymbol{x}_{k}+\boldsymbol{t}^{-}, \boldsymbol{y}_{k}-\boldsymbol{t}^{+} ; \boldsymbol{g}^{I}, \boldsymbol{g}^{O}\right) \geq \beta_{k}^{*}=B D D F_{C R S}\left(\boldsymbol{x}_{k}, \boldsymbol{y}_{k} ; \boldsymbol{g}^{I}, \boldsymbol{g}^{O}\right)$. 
(b) It is a direct consequence of the units invariant property of the DDF (unbounded) models (see Färe and Grosskopf 2010, p. 321).

Proposition 5 establishes that, the new measure is weakly monotonic in the case of using a constant directional vector, independent of the point being rated. Moreover, the measure is units invariant if the directional vector is expressed in the units of the data, for example if $\boldsymbol{g}_{k}=\left(\boldsymbol{x}_{k}, \boldsymbol{y}_{k}\right)$.

\section{The partially bounded models}

In any DEA model we may consider variables that are naturally bounded. For instance, if a variable is expressed as a percentage and is considered as an output, the "natural" upper bound would be 100. Additionally, if the variable is considered an input, the upper bound does not play any role and we can ignore it. A similar statement is valid for ratio-variables. Some inputs may have lower bounds in a production process if, for economical or technological reasons, it makes no sense to consider a production unit that does not consume a minimum of some of the inputs. Let us assume that only the first $m_{1} \leq m$ inputs are lower bounded and, similarly, that only the first $s_{1} \leq s$ outputs are upper bounded. It is straightforward to formulate the corresponding CRS partially-bounded technology, $T^{P B}$, that gives an expression similar to (5).

The formulation of the corresponding model follows.

$$
\begin{aligned}
& \operatorname{PBDDF}_{C R S}\left(\boldsymbol{x}_{k}, \boldsymbol{y}_{k} ; \boldsymbol{g}_{k}^{I}, \boldsymbol{g}_{k}^{O}\right)=\operatorname{Max} \beta_{k}^{P} \\
& \text { s.t. } \\
& \begin{array}{llrl}
\sum_{j \in E} \lambda_{j k} x_{i j} \leq x_{i k}-\beta_{k}^{P} g_{i k}^{I}, & i & =1, \ldots, m \\
\sum_{j \in E} \lambda_{j k} y_{r j} \geq y_{r k}+\beta_{k}^{P} g_{r k}^{O}, x_{i k}-\beta_{k}^{P} g_{i k}^{I} \geq \underline{x}_{i}, & r=1, \ldots, s
\end{array} \\
& x_{i k}-\beta_{k}^{P} g_{i k}^{I} \geq \underline{x}_{i}, \quad i=1, \ldots, m_{1} \\
& y_{r k}+\beta_{k}^{P} g_{r k}^{O} \leq \bar{y}_{r}, \\
& \lambda_{j k} \geq 0 \text {, } \\
& \beta_{k}^{P} \geq 0 \\
& r=1, \ldots, s_{1} \\
& j \in E
\end{aligned}
$$

It can be proved that the CRS Partially-bounded DDF model inherits the same properties as the Bounded CRS-DDF model. We will list them directly.

Proposition 6 The Partially-bounded DDF inefficiency measure satisfies the following properties.

(a) If PBDDF $F_{C R S}\left(\boldsymbol{x}_{k}, \boldsymbol{y}_{k} ; \boldsymbol{g}_{k}^{I}, \boldsymbol{g}_{k}^{O}\right)=0$ then $\left(\boldsymbol{x}_{k}, \boldsymbol{y}_{k}\right) \in \partial^{w}\left(T^{P B}\right)$.

(b) $P B D D F_{C R S}\left(\boldsymbol{x}_{k}, \boldsymbol{y}_{k} ; \boldsymbol{g}_{k}^{I}, \boldsymbol{g}_{k}^{O}\right)$ is weakly monotonic, as long as the directional vector is a constant vector.

(c) $P B D D F_{C R S}\left(\boldsymbol{x}_{k}, \boldsymbol{y}_{k} ; \boldsymbol{g}_{k}^{I}, \boldsymbol{g}_{k}^{O}\right)$ is units invariant, as long as each component of the directional vector is expressed in the same units of measurement as the corresponding input or output. 


\section{Productivity change measurement}

The measurement of productivity change over time using frontier methods continues to claim considerable attention in the literature that focuses on the assessment of economic performance of decision making units. The most popular approach to evaluate productivity change is the Malmquist productivity index introduced by Caves et al. (1982) and decomposed for the first time by Färe et al. (1992) into efficiency and technical changes. The Malmquist index is a ratio-based index that uses the Shephard (1953) distance functions to evaluate each unit under scrutiny and, in its most popular forms, adopt either an input contraction or an output expansion perspective. Malmquist indexes are only appropriate for dealing with efficiency or inverse efficiency measures with values in the range $[0,1]$ and belong to the "multiplicative world". They are not suitable for dealing with inefficiency measures as the directional distance function. For this reason, Chambers et al. (1996b) introduced the Luenberger indicator, which is a difference-based index of directional distance functions that can account for input contractions, output improvements, or both. In contrast to the Malmquist index, Luenberger indicators belong to the "additive world".

Once we have introduced the Bounded CRS Directional Distance Function and its Partially-bounded version in the paper, we will go on to show in this section how they can be used in order to estimate productivity change and its components over time. This is one of the interesting applications of the bounded DDF model under constant returns to scale. Its operation is completely similar to the usual DDF model.

In a context where measuring productivity is the focus, assuming constant returns to scale for DEA models is a must. This viewpoint has been followed since the papers by Grifell-Tatjé and Lovell (1995), Ray and Desli (1997), Balk (2001) and Lovell (2003) for the Malmquist productivity index and, likewise, for the Luenberger productivity indicator since its definition (see, for example, a recent application that follows this philosophy in Kapelko et al. 2015). However, at the present time this assumption wouldn't seem to fit well with datasets where one or more variables (inputs and outputs) are bounded. In the absence of appropriate Bounded CRS DEA models, the traditional literature supports the use of the hypothesis of Variable Returns to Scale instead (Hollingsworth and Smith 2003), simply because it guarantees that the efficient projections points have, for example for a percentage variable, an upper-bounded value, lower or equal to 100. In contrast, the hereby introduced new Bounded or Partiallybounded CRS Directional Distance Functions allow to combine productivity change measurement based on CRS models and deal with ratio or percentage variables or, in general, bounded inputs and outputs in a natural way.

Before showing the formulation of the Luenberger productivity indicator, we need to introduce new notation, associated with time periods. Let us suppose that we have observed a sample of $n$ units for $p$ different periods of time. Unit $j \in\{1,2, \ldots, n\}$ in period $t \in\{1,2, \ldots, p\}$ uses a specific amount of $m$ inputs, $\boldsymbol{x}_{j}^{t}=\left(x_{1 j}^{t}, \ldots, x_{m j}^{t}\right) \in$ $R_{++}^{m}$, to produce a certain amount of $s$ outputs $\boldsymbol{y}_{j}^{t}=\left(y_{1 j}^{t}, \ldots, y_{s j}^{t}\right) \in R_{++}^{s}$. Moreover, seeking simplicity, let us also develop the Luenberger indicator for the case of using Partially-bounded CRS Directional Distance Functions. To do that, we need to adapt model (11) in order to perform an intertemporal analysis. Suppose that we want to 
evaluate unit $k, k=1, \ldots, n$, observed in period $d, d=1, \ldots, p$, with respect to the production possibility set related to period $h, h=1, \ldots, p$. The $d$ and $h$ indices may or may not coincide. Additionally, $\underline{x}_{i}^{h}$ denotes the lower bound of input $i, i=1, \ldots, m_{1}$, $\bar{y}_{r}^{h}$ denotes the upper bound of output $r, r=1, \ldots, s_{1}$, and $E^{h}$ denotes the set of Pareto-efficient DMUs in period $h$.

$$
\begin{aligned}
& P B D D F_{C R S}^{h}\left(\boldsymbol{x}_{k}^{d}, \boldsymbol{y}_{k}^{d} ; \boldsymbol{g}_{k}^{I d}, \boldsymbol{g}_{k}^{O d}\right)=\operatorname{Max} \beta_{k}^{h} \\
& \text { s.t. } \\
& \sum_{j \in E^{h}} \lambda_{j k} x_{i j}^{h} \leq x_{i k}^{d}-\beta_{k}^{h} g_{i k}^{I d}, i=1, \ldots, m \\
& \sum_{j \in E^{h}} \lambda_{j k} y_{r j}^{h} \geq y_{r k}^{d}+\beta_{k}^{h} g_{r k}^{O d}, r=1, \ldots, s \\
& x_{i k}^{d}-\beta_{k}^{h} g_{i k}^{I d} \geq \underline{x}_{i}^{h}, \quad i=1, \ldots, m_{1} \\
& y_{r k}^{d}+\beta_{k}^{h} g_{r k}^{O d} \leq \bar{y}_{r}^{h}, \quad r=1, \ldots, s_{1} \\
& \lambda_{j k} \geq 0 \text {, } \\
& \beta_{k}^{h} \text { free }
\end{aligned}
$$

Note that in (12) the decision variable $\beta_{k}^{h}$ is not restricted in sign, allowing it to take both non-negative and negative values. Observe also that $m_{1}=m$ and $s_{1}=s$ corresponds to the case where all the inputs are lower bounded and all the outputs are upper bounded. In this case we will simply write $B D D F_{C R S}^{h}\left(\boldsymbol{x}_{k}^{d}, \boldsymbol{y}_{k}^{d} ; \boldsymbol{g}_{k}^{I d}, \boldsymbol{g}_{k}^{O d}\right)$ deleting the initial $P$. As happens in the case of the traditional directional distance functions (see Chambers et al. 1998), a negative value for $\beta_{k}^{h *}$ will be associated with an evaluated unit located outside the reference technology, while a non-negative value will imply that the assessed unit is within the production possibility set.

Now we are ready to measure productivity change resorting to a Luenberger indicator (Chambers et al. 1996b) and decompose it into its two usual components. First of all, the (total factor) productivity change for unit $k$ when comparing periods $t$ and $t+1$ is measured by means of:

$$
\begin{aligned}
T F P_{k}(t, t+1)= & \frac{1}{2}\left[P B D D F_{C R S}^{t}\left(\boldsymbol{x}_{k}^{t}, \boldsymbol{y}_{k}^{t} ; \boldsymbol{g}_{k}^{I t}, \boldsymbol{g}_{k}^{O t}\right)-P B D D F_{C R S}^{t}\left(\boldsymbol{x}_{k}^{t+1}, \boldsymbol{y}_{k}^{t+1} ; \boldsymbol{g}_{k}^{I t+1}, \boldsymbol{g}_{k}^{O t+1}\right)\right]+ \\
& +\frac{1}{2}\left[P B D D F_{C R S}^{t+1}\left(\boldsymbol{x}_{k}^{t}, \boldsymbol{y}_{k}^{t} ; \boldsymbol{g}_{k}^{I t}, \boldsymbol{g}_{k}^{O t}\right)-P B D D F_{C R S}^{t+1}\left(\boldsymbol{x}_{k}^{t+1}, \boldsymbol{y}_{k}^{t+1} ; \boldsymbol{g}_{k}^{I t+1}, \boldsymbol{g}_{k}^{O t+1}\right)\right],
\end{aligned}
$$

As usual, the Luenberger indicator may then be decomposed into technological change (TC) and efficiency change (EC).

$$
\begin{aligned}
T C_{k}(t, t+1)= & \frac{1}{2}\left[P B D D F_{C R S}^{t+1}\left(\boldsymbol{x}_{k}^{t+1}, \boldsymbol{y}_{k}^{t+1} ; \boldsymbol{g}_{k}^{I t+1}, \boldsymbol{g}_{k}^{O t+1}\right)-P B D D F_{C R S}^{t}\left(\boldsymbol{x}_{k}^{t+1}, \boldsymbol{y}_{k}^{t+1} ; \boldsymbol{g}_{k}^{I t+1}, \boldsymbol{g}_{k}^{O t+1}\right)\right] \\
& +\frac{1}{2}\left[P B D D F_{C R S}^{t+1}\left(\boldsymbol{x}_{k}^{t}, \boldsymbol{y}_{k}^{t} ; \boldsymbol{g}_{k}^{I t}, \boldsymbol{g}_{k}^{O t}\right)-P B D D F_{C R S}^{t}\left(\boldsymbol{x}_{k}^{t}, \boldsymbol{y}_{k}^{t} ; \boldsymbol{g}_{k}^{I t}, \boldsymbol{g}_{k}^{O t}\right)\right] . \\
E C_{k}(t, t+1) & =P B D D F_{C R S}^{t}\left(\boldsymbol{x}_{k}^{t}, \boldsymbol{y}_{k}^{t} ; \boldsymbol{g}_{k}^{I t}, \boldsymbol{g}_{k}^{O t}\right)-P B D D F_{C R S}^{t+1}\left(\boldsymbol{x}_{k}^{t+1}, \boldsymbol{y}_{k}^{t+1} ; \boldsymbol{g}_{k}^{I t+1}, \boldsymbol{g}_{k}^{O t+1}\right) .
\end{aligned}
$$

We illustrate the new methodology by an empirical example in the next section. 


\section{An empirical example}

We have gathered public agricultural data from the Spanish Ministry of "Agriculture, Land and Food" relative to virgin olive oil producers (MAGRAMA 2016). As it is well known, olive oil is one of the basic ingredients of the so called Mediterranean diet. In fact, $97 \%$ of the world production corresponds to Mediterranean countries, where Spain, Italy and Greece account for $75 \%$ of the mentioned production and Spain is the largest producer (50\%). Without question, one of the most successful formulas that agri-food operators have when it comes to competing in global markets consists of emphasizing all the quality aspects related to the provenance of products through different figures of protection. One of these figures is precisely the Protected Designation of Origin (PDO). Moreover, the type of oil produced is regulated by the European Commission (through EC regulation 1234/2007). The two best qualities are grouped under the name "virgin olive oil". Being more precise we have selected all the PDOs with available data for two consecutive years $(2012,2013)$. They comprise 22 of the 29 existing PDOs in Spain. Their names and acronyms are listed in Table 1.

From MAGRAMA (2016), we got information corresponding to the following 'absolute' data related to each PDO in each of the two considered years:

Ha: surface devoted to olive farming in thousands of hectares

Bott: the number of bottlers ${ }^{3}$

POO: the number of tons of any kind of olive oil produced and sold ${ }^{4}$

VOO: the number of tons of virgin olive oil produced and sold ${ }^{5}$

Rev: the total revenue obtained from POO expressed in millions of euros

The above variables are not directly the inputs and outputs that we will use in the empirical application. Instead, in our model, Ha and Bott will be the inputs, while we will consider only two outputs: Rev and the percentage of VOO over POO (VOO/POO \%). The rationale behind the use of $\mathrm{VOO} / \mathrm{POO} \%$ is the following. The main objective of virgin olive oil PDOs is to produce, certify and commercialize virgin oil, being this the highest quality olive oil and the one that provides more health benefits, in addition to achieve the highest prices in the market. Hence, a key objective is to maximize the production of this kind of oil over others, ensuring the highest profitability. However not all the oil produced by the PDO can be qualified as virgin oil for various reasons. There are legally bindings by Regulatory Boards of the PDOs, for example those concerning the variety or varieties that should be certified, the production techniques (kind of pressing, harvest, etc.) or the minimum quality standards (acidity level, bouquet, etc.). Moreover, weather plays an important role in agriculture. In particular, excess rainfall or, by contrast, long dry periods, solar radiation or certain pests and diseases (Bactrocera - Dacus oleae -, Phoeotribus scarabeoides, etc) impact on the quality and, hence, on the impossibility of certain oils to be qualified as virgin.

\footnotetext{
${ }^{3}$ In our empirical context, the bottlers are also marketers.

${ }^{4}$ Since olive oil is a perishable product and must be consumed during its first year of life, all the yearly production must be sold.

5 This is the part of the production of olive oil with the highest quality, either extra virgin olive oil or virgin olive oil.
} 
Table 1 Names and acronyms of the 22 considered PDOs

\begin{tabular}{lll}
\hline$\#$ & PDO & Acronym \\
\hline 1 & Campo de Montiel & C Montiel \\
& Oil & \\
3 & La Alcarria Oil & Alcarria \\
& Oil from the & CV \\
4 & Valencian Region & \\
5 & Oil from Majorca & Mallorca \\
6 & Terra Alta Oil & Terra Alta \\
& Oil from Baix & B Ebre-Mo \\
7 & Ebre-Montsià & \\
& Oil from Lower & B Aragon \\
8 & Aragón & \\
9 & Monterrubio Oil & Mterrubio \\
10 & Antequera & Antequera \\
11 & Baena & Baena \\
12 & Estepa & Estepa \\
13 & Les Garrigues & L Garrigues \\
14 & Montes de Granada & M Granada \\
15 & Montes de Toledo & M Toledo \\
16 & Montoro-Adamuz & M-Adamuz \\
17 & Poniente de Granada & P Granada \\
18 & Priego de Córdoba & Priego Cor \\
19 & Sierra de Cádiz & S Cadiz \\
20 & Sierra de Cazorla & S Cazorla \\
21 & Sierra de Segura & S Segura \\
22 & Sierra Mágina & S Magina \\
& Siurana & Siurana \\
\hline & & \\
& &
\end{tabular}

Table 2 Summary statistics of inputs and outputs, year 2012

\begin{tabular}{lrrcr}
\hline & INPUT 1 Ha & INPUT 2 Bott & $\begin{array}{l}\text { OUTPUT 1 } \\
\text { VOO/POO\% }\end{array}$ & OUTPUT 2 Rev \\
\hline Max & 61.00 & 40.00 & 100.00 & 14.66 \\
Min & 1.81 & 1.00 & 3.00 & 0.01 \\
Avg & 26.11 & 13.32 & 55.32 & 4.62 \\
SD & 16.03 & 9.88 & 42.38 & 5.23 \\
\hline
\end{tabular}

According to an economic and productive logic, the target of each PDO is to maximize the VOO/POO ratio, since it means that all the olive oil produced has been virgin oil, which represents the highest quality level, fulfilling all the standards established by PDO's managers and resulting in the highest market value.

A statistical summary, for each year, of the selected variables are presented in Tables 2 and 3 . 
Table 3 Summary statistics of inputs and outputs, year 2013

\begin{tabular}{lrrcc}
\hline & INPUT 1 Ha & INPUT 2 Bott & $\begin{array}{l}\text { OUTPUT 1 } \\
\text { VOO/POO } \%\end{array}$ & OUTPUT 2 Rev \\
\hline Max & 60.00 & 40.00 & 100.00 & 18.34 \\
Min & 1.78 & 1.00 & 6.00 & 0.03 \\
Avg & 26.44 & 13.41 & 66.27 & 4.73 \\
SD & 15.89 & 11.16 & 39.26 & 5.29 \\
\hline
\end{tabular}

Comparing Tables 2 and 3 we appreciate that, on average, variables have changed slightly, with the exception of $\mathrm{VOO} / \mathrm{POO} \%$ where the percentage of virgin olive oil sold over the total olive oil sold has increased over $10 \%$ points. Revising the maximums, only Rev has changed significantly, with an increase of $25 \%$ in revenue, while the minimums have only changed significantly at $\mathrm{VOO} / \mathrm{POO} \%$. Finally, the standard deviation has a moderate change in Rev, Bott, and in VOO/POO\%.

If now we eliminate the size of each PDO and obtain a sample of PDOs with the same size, it is clear that we need to resort to a CRS model since it allows a fair comparison between all the PDOs. This can be accomplished by dividing Bott and Rev by Ha since VOO/POO\% already coincides with the ratio between $\mathrm{VOO} / \mathrm{Ha}$ and POO/Ha. As Hollingsworth and Smith (2003, p. 734) pointed out, if we resort to a CCR output oriented model (Charnes et al. 1978), the same results will be obtained using the original inputs and outputs (Ha, Bott, Rev and VOO/POO\%) or with the transformed variables Bott/ $\mathrm{Ha}$, Rev/Ha and $\mathrm{VOO} / \mathrm{POO} \%$. In our case, we will consider a CRS DDF model with directional vector $\boldsymbol{g}_{k}=\left(\boldsymbol{0}_{m}, \boldsymbol{y}_{k}\right)$, which is equivalent to a CCR output oriented model, as Chambers et al. (1998) proved. Given that result of equivalence, we will consider the original inputs and outputs (Ha, Bott, Rev and VOO/POO\%) in the model and use a CRS DDF with a "proportional" output-oriented directional vector, with the only particularity that one of the outputs is a natural upper bounded variable (VOO/POO\%). We will finally consider a Partially-Bounded CRS DDF model with a single upper-bounded output, the variable being expressed as a percentage. ${ }^{6}$

In order to set up model (11) we need first to identify the CRS strongly efficient units of the two considered frontiers corresponding to years 2012 and 2013. Resorting to the CRS additive model (Ali and Seiford 1993), for 2012 we obtain six strongly efficient units (U2-Alcarria, U4-Mallorca, U7-B. Aragon, U11—Estepa, U17—Priego

\footnotetext{
6 Our empirical application and that published in Vidal et al. (2014) are different regarding the data because Vidal et al. (2014) used the available information of the years 2008, 2009 and 2010 for 17 DMUs, while we utilize data from 2012 and 2013 for 22 DMUs. The inputs are similar but the outputs are more different. Vidal et al. (2014) did not use Revenue as output. They also did not utilize the ratio VOO/POO, which measures the 'quality' of the produced outputs. Instead, Vidal et al. (2014) directly used tons of olive oil. In Vidal et al. (2014), none of the variables is bounded in a natural way and were really bounded by the minimum observed input and maximum observed output. Additionally, from a methodological point of view, these approaches are so different since (1) Vidal et al. (2014) resorted to the 'Graph' version of a BAM, which are based upon the Weighted Additive Model, bounding all inputs and outputs, whereas our approach is based on an output-oriented (Bounded) DDF, where also only one dimension is bounded; and (2) the objective in Vidal et al. (2014) was to measure technical efficiency and its evolution, while we also determined productivity change over time and its drivers.
} 
Table 4 Comparing the unbounded and the partially-bounded DDF model, year 2012

\begin{tabular}{lccc}
\hline & $\operatorname{DDF}^{12}(12)$ & $\operatorname{PBDDF}^{12}(12)$ & Difference \\
\hline Max & 39.86 & 24.00 & 15.86 \\
Min & 0.00 & 0.00 & 0.00 \\
Avg & 3.84 & 2.74 & 1.09 \\
SD & 8.59 & 5.46 & 3.13 \\
\hline
\end{tabular}

Cor, and U22 - Siurana), while five strongly efficient units were identified for 2013 (U4, U7, U8-Mterrubio, U11 and U22). As can be detected, four strongly efficient units from 2012 remain as such in 2013. Hence, $E^{2012}=\{\mathrm{U} 2, \mathrm{U} 4, \mathrm{U} 7, \mathrm{U11}, \mathrm{U} 17, \mathrm{U} 22\}$ and $E^{2013}=\{\mathrm{U} 2, \mathrm{U} 4, \mathrm{U} 7, \mathrm{U} 8, \mathrm{U} 22\}$. Before starting the productivity analysis, let us show in this experimental case the influence, if any, of the considered bounds. We just focus on the year 2012, i.e., the frontier of year 2012 as well as the 22 units of the same year, and run the traditional DDF model without bounds and our new model with a single natural bound for $\mathrm{VOO} / \mathrm{POO} \%$. Since the latter model has more restrictions, the expected objective function value for each unit will be smaller than or equal to that corresponding to the unbounded model. In Table 4 we provide a summary comparison about the optimal value of both models, adding the information about the difference between both models in the last column.

The main conclusion is that there are relevant differences. For instance, the maximum reduction is close to $40 \%$ points, while the average reduction is slightly over $28 \%$ points. Meanwhile, the standard deviation has decreased over 36\% points. Looking at the individual units, the 6 strongly efficient units cannot change their status; hence, only 16 units can modify its optimal value. The majority of units have changed (U1, $\mathrm{U} 3, \mathrm{U} 5, \mathrm{U} 12, \mathrm{U} 13, \mathrm{U} 14, \mathrm{U16}, \mathrm{U18}, \mathrm{U} 20$ ). We will not carry on this simple exercise but the conclusion is straightforward: the presence of bounds does influence the efficiency evaluation, and, as a direct consequence, the productivity analyses.

Let us now undertake the productivity analysis. The first step is to calculate the total factor productivity change at each $\mathrm{PDO}_{k}, T F P_{k}(t, t+1), k=1, \ldots, 22, t=2012$. According to expression (13) we need to project each of the 44 PDOs, corresponding to years 2012 and 2013, onto each of the corresponding frontiers, resorting to the selected PBDDF. The only considered bound for VOO/POO $\%$ is 100 . The results obtained are listed in Table 5. In each of the last four columns the header is $\operatorname{PBDDF}^{\mathrm{h}}(\mathrm{d})$, where the superscript stands for the year of the frontier (12 or 13) and d stands for the year of the projected data.

As expected, the result for the projection of any unit onto its own frontier is always nonnegative (see columns 3 and 6 in Table 5). In column 3, the projection of the 2012 units onto its own frontier shows that as many as 12 units are projected onto the frontier, getting a 0 optimal value, which, of course, include the six already identified strongly efficient units. The other six units may or may not belong to the strongly efficient frontier. Similarly, in the year 2013 (see column 6), 12 units also get a 0 value. Ten units are projected, at the same time, onto the 2012 and 2013 frontier. In columns 4 and 5 the output inefficiency is reported for the projection of 
Table 5 Data projections for the years 2012 and 2013 onto each of the two yearly frontiers

\begin{tabular}{llcccl}
\hline$\#$ & PDO & PBDDF $^{12}(12)$ & $\operatorname{PBDDF}^{12}(13)$ & $\operatorname{PBDDF}^{13}(12)$ & $\operatorname{PBDDF}^{13}(13)$ \\
\hline 1 & C Montiel & 0.00 & 0.00 & 0.00 & 0.00 \\
2 & Alcarria & 0.00 & 12.34 & -0.50 & 7.33 \\
3 & CV & 0.00 & 0.00 & 0.00 & 0.00 \\
4 & Mallorca & 0.00 & -0.18 & -0.12 & 0.00 \\
5 & Terra Alta & 0.00 & 0.00 & 0.00 & 0.00 \\
6 & B Ebre-Mo & 0.73 & -0.26 & 1.33 & 0.00 \\
7 & B Aragon & 0.00 & -0.05 & 0.00 & 0.00 \\
8 & Mterrubio & 2.35 & -0.53 & 4.76 & 0.00 \\
9 & Antequera & 6.88 & 0.11 & 5.89 & 0.03 \\
10 & Baena & 1.87 & 0.76 & 1.15 & 0.32 \\
11 & Estepa & 0.00 & 0.18 & -0.22 & 0.00 \\
12 & L Garrigues & 0.00 & 0.00 & 0.00 & 0.00 \\
13 & M Granada & 24.00 & 4.56 & 24.00 & 4.56 \\
14 & M Toledo & 0.00 & 0.00 & 0.00 & 0.00 \\
15 & M-Adamuz & 8.53 & 2.63 & 7.07 & 2.28 \\
16 & P Granada & 0.00 & 0.00 & 0.00 & 0.00 \\
17 & Priego Cor & 0.00 & 0.28 & -0.25 & 0.28 \\
18 & S Cadiz & 6.69 & 6.69 & 6.69 & 6.69 \\
19 & S Cazorla & 0.27 & 0.54 & 0.05 & 0.26 \\
20 & S Segura & 4.56 & 9.01 & 4.56 & 7.01 \\
21 & S Magina & 4.51 & 0.82 & 3.22 & 0.82 \\
22 & Siurana & 0.00 & -0.29 & 0.37 & 0.00 \\
\hline & & & &
\end{tabular}

each unit onto the other year's frontier. The few obtained negative optimal values have got a italic background. Only 5 units of the year 2013 do not belong to year 2012's production possibility set (see column 4), while only 4 units of year 2012 do not belong to year 2013's production possibility set (see column 5). There is a clear overlap between parts of the two yearly frontiers, which means that, on average, we cannot expect a relevant productivity change between the two considered years.

The Luenberger total factor productivity change as well as its two additive components, technical change and efficiency change are reported for each PDO in Table 6. Two units present the two extreme behaviors in our sample, namely U2, Alcarria and U13, M-Granada. The first one shows a very negative evolution from 2012 to 2013 , with a productivity change of -10.09 , decomposed into an efficiency change of -7.33 and a technical change of -2.75 . On the other hand, U13 shows a very positive evolution from 2012 to 2013, with a productivity change of 19.44 that is all attributable to efficiency change. In agriculture, these high productivity changes have an easy explanation. In the first case, Alcarria, the harvest of olives in 2013 was seriously reduced in comparison to the year 2012, due basically to adverse weather 
Table 6 Luenberger productivity results for each PDO

\begin{tabular}{|c|c|c|c|c|}
\hline \# & PDO & TFP & $\mathrm{EC}$ & $\mathrm{TC}$ \\
\hline 1 & C Montiel & 0.00 & 0.00 & 0.00 \\
\hline 2 & Alcarria & -10.09 & -7.33 & -2.75 \\
\hline 3 & $\mathrm{CV}$ & 0.00 & 0.00 & 0.00 \\
\hline 4 & Mallorca & 0.03 & 0.00 & 0.03 \\
\hline 5 & Terra Alta & 0.00 & 0.00 & 0.00 \\
\hline 6 & B Ebre-Mo & 1.16 & 0.73 & 0.43 \\
\hline 7 & B Aragon & 0.02 & 0.00 & 0.02 \\
\hline 8 & Mterrubio & 3.82 & 2.35 & 1.47 \\
\hline 9 & Antequera & 6.31 & 6.85 & -0.54 \\
\hline 10 & Baena & 0.97 & 1.55 & -0.58 \\
\hline 11 & Estepa & -0.20 & 0.00 & -0.20 \\
\hline 12 & L Garrigues & 0.00 & 0.00 & 0.00 \\
\hline 13 & M Granada & 19.44 & 19.44 & 0.00 \\
\hline 14 & M Toledo & 0.00 & 0.00 & 0.00 \\
\hline 15 & M-Adamuz & 5.34 & 6.24 & -0.90 \\
\hline 16 & P Granada & 0.00 & 0.00 & 0.00 \\
\hline 17 & Priego Cor & -0.41 & -0.28 & -0.13 \\
\hline 18 & S Cadiz & 0.00 & 0.00 & 0.00 \\
\hline 19 & S Cazorla & -0.24 & 0.02 & -0.26 \\
\hline 20 & S Segura & -3.45 & -2.46 & -1.00 \\
\hline 21 & S Magina & 3.05 & 3.69 & -0.64 \\
\hline 22 & Siurana & 0.33 & 0.00 & 0.33 \\
\hline
\end{tabular}

conditions. On the other hand, in M-Granada the misfortune was located in 2012 and the olive harvest recovered strongly in 2013. Besides these two extreme PDOs, and the intense productivity progress of units U9, Antequera, and U15, M-Ademuz, the rest of PDOs have a notable or moderate productivity progress or regress, in the range $[-3.45,3.82]$. In fact there is a subset of 7 PDOs, $\{\mathrm{U} 1, \mathrm{U} 3, \mathrm{U} 5, \mathrm{U} 12, \mathrm{U} 14$, U16, U18\}, which experience no change at all and another subset of 4 PDOs, \{U4, U7, U11, U22 \}, with an imperceptible productivity progress, or, in the case of U11, regress. The strongly efficient units that were identified as points of the two efficient frontiers, U4, U7, U11 and U22, belong to one of the two last considered subsets. The two strongly efficient units that belong to the 2012 frontier but not to the 2013 frontier, U2 and U17, experience a productivity regress, while the only strongly efficient unit that belongs only to the 2013 frontier, U8, experiences productivity progress.

Finally, Table 7 presents a summary of the productivity results. The most relevant issue is the average behavior, which shows a moderate productivity progress that is completely due to the catching up effect—or positive efficiency change—accompanied by a little negative technical change. 
Table 7 Productivity results summary

\begin{tabular}{lrrr}
\hline $2012-2013$ & \multicolumn{1}{c}{ TFP } & \multicolumn{1}{c}{ EC } & \multicolumn{1}{c}{ TC } \\
\hline Max & 19.44 & 19.44 & 1.47 \\
Min & -10.09 & -7.33 & -2.75 \\
Avg & 1.19 & 1.40 & -0.21 \\
SD & 5.14 & 4.89 & 0.76 \\
\hline
\end{tabular}

\section{Conclusions}

We have been able to extend the use of bounds, initially proposed exclusively for additive models, to the family of Directional Distance Function models. The VRS models are all naturally bounded models, with the range bounds associated to the finite experimental sample, acting as lower bounds for the inputs and as upper bounds for the outputs and can be a good choice unless the nature of the available inputs and outputs determines that the different units under comparison can be judged on an equal footing. In that case, resorting to a CRS model is a must. Moreover, the nature of the considered variables establishes the need to resort to a specific bounded model. This has been the case with our olive oil production empirical case developed in Sect. 6, where one of the outputs was a percentage that has associated the value 100 as a natural upper bound. In other cases, for example, when the considered sample corresponds to the whole population, the range bounds are the appropriate choice for any considered Bounded CRS model. We have focused our attention on the Directional Distance Function model because, being one of the most widely used models in DEA that measures technical inefficiency, the use of bounds has been ignored.

In almost all the practical cases where bounds play a role, we note that not all the variables, inputs and outputs, have to be necessarily bounded. Accordingly, we have also defined the partially-bounded CRS-DDF models, where the introduced natural bounds will avoid the projection of any inefficient units towards a point that is unacceptable, because it does not satisfy the corresponding natural bounds. This has been the case in our empirical application in the context of productivity change estimation over time, where, as explained in the preceding sections, the assumption of constant returns to scale is inevitable. So far, this combination was unachievable in the literature. The Bounded or Partially-bounded CRS Directional Distance Function, combined with the corresponding Luenberger indicator, permits these types of estimations to be carried out, as shown theoretically in Sect. 5 and empirically in Sect. 6.

Acknowledgements We thank the guest editors of the special issue DEA 2017 and two anonymous referees for providing constructive comments and help in improving the contents and presentation of this paper. Additionally, J.T. Pastor, J. Aparicio, J. Alcaraz and F. Vidal thank the financial support from the Spanish Ministry for Economy and Competitiveness (Ministerio de Economía, Industria y Competitividad), the State Research Agency (Agencia Estatal de Investigacion) and the European Regional Development Fund (Fondo Europeo de DEsarrollo Regional) under Grant MTM2016-79765-P (AEI/FEDER, UE). 


\section{References}

Ali AI, Seiford LM (1993) The mathematical programming approach to efficiency analysis. In: Fried H, Lovell CAK, Schmidt SS (eds) The measurement of productive efficiency: techniques and applications. Oxford University Press, Inc, Oxford

Balk B (2001) Scale efficiency and productivity change. J Prod Anal 15:159-183

Briec W, Kerstens K (2009a) Infeasibility and directional distance functions with application of determinateness of the Luenberger productivity indicator. J Optim Theory Appl 141:55-73

Briec W, Kerstens K (2009b) The Luenberger productivity indicator: an economic specification leading to infeasibilities. Econ Model 26:597-600

Caves DW, Christensen LR, Diewert WE (1982) The economic theory of index numbers and the measurement of input, output, and productivity. Econometrica 50:1393-1414

Chambers RG, Chung Y, Färe R (1996a) Benefit and distance functions. J Econ Theory 70:407-419

Chambers RG, Färe R, Grosskopf S (1996b) Productivity growth in APEC countries. Pac Econ Rev 1:181-190

Chambers RG, Chung Y, Färe R (1998) Profit, directional distance functions, and Nerlovian efficiency. J Optim Theory Appl 98(2):351-364

Charnes A, Cooper WW, Rhodes E (1978) Measuring the efficiency of decision making units. Eur J Oper Res 2:429-444

Cooper WW, Park KS, Pastor JT (1999) RAM: a range adjusted measure of inefficiency for use with additive models, and relations to others models and measures in DEA. J Prod Anal 11:5-42

Cooper WW, Pastor JT, Borras F, Aparicio J, Pastor D (2011) BAM: a bounded adjusted measure of efficiency for use with bounded additive models. J Prod Anal 35(2):85-94

Färe R, Grosskopf S (2010) Directional distance functions and slacks-based measures of efficiency. Eur J Oper Res 200:320-322

Färe R, Grosskopf S, Lindgren B, Roos P (1992) Productivity changes in swedish pharmacies 1980-1989: a non-parametric malmquist approach. J Prod Anal 3:85-101

Grifell-Tatjé E, Lovell CAK (1995) A note on the malmquist productivity index. Econ Lett 47:169-175

Hollingsworth B, Smith P (2003) Use of ratios in data envelopment analysis. Appl Econ Lett 10:733-735

Kapelko M, Horta IM, Camanho AS, Oude Lansink A (2015) Measurement of input specific productivity growth with an application to the construction industry in Spain and Portugal. Int J Prod Econ 166:64-71

Koopmans TC (1951) Analysis of production as an efficient combination of activities. In: Koopmans TC (ed) Activity analysis of production and allocation. Wiley, New York

Liu JS, Lu LYY, Lu W-M, Lin BJY (2013) Data envelopment analysis 1978-2010: a citation-based literature survey. Omega 41:3-15

Lovell CAK (2003) The decomposition of Malmquist productivity indexes. J Prod Anal 20:437-458

Luenberger DG (1992) New optimality principles for economic efficiency and equilibrium. J Optim Theory Appl 75(2):221-264

MAGRAMA (2016) Estadisticas. Producciones Agricolas. Ministerio de Agricultura, Alimentacion y Medio Ambiente. http://www.magrama.gob.es/es/agricultura/estadisticas/. Accessed 28 Dec 2016 (in Spanish)

Olesen OB, Petersen NC, Podinovski VV (2015) Efficiency analysis with ratio measures. Eur J Oper Res 245(2):446-462

Pastor JT, Aparicio J, Monge JF, Pastor D (2013) Modeling CRS bounded additive DEA models and characterizing their Pareto-efficient points. J Prod Anal 40:285-292

Pastor JT, Del Campo FJ, Vidal F, Pastor D (2014) Efficiency in attracting tourists via the Web-an application to the Mediterranean countries. Tour Econ 20(1):195-202

Pastor JT, Aparicio J, Alcaraz J, Vidal F, Pastor D (2015) An enhanced BAM for unbounded or partially bounded CRS additive models. Omega 56:16-24

Rashidi K, Saen RF (2015) Measuring eco-efficiency based on green indicators and potentials in energy saving and undesirable output abatement. Energy Econ 50:18-26

Ray SC, Desli E (1997) Productivity growth, technical progress, and efficiency change in industrialized countries. Am Econ Rev 87:1033-1039

Shephard RW (1953) Cost and production functions. Princeton University Press, Princeton 
Toloo M, Tavana M, Santos-Arteaga FJ (2017) An integrated data envelopment analysis and mixed integer non-linear programming model for linearizing the common set of weights. CEJOR. https://doi.org/1 0.1007/s10100-017-0510-y

Toloo M, Nalchigar S, Sohrabi B (2018) Selecting most efficient information system projects in presence of user subjective opinions: a DEA approach. CEJOR. https://doi.org/10.1007/s10100-018-0549-4

Vidal F, Pastor JT, Borras F, Pastor D (2013) Efficiency analysis of the designations of origin in the Spanish wine sector. Span J Agri Res 11(2):294-304

Vidal F, Aparicio J, Pastor JT, Pastor D (2014) Las Denominaciones de Origen de aceite de oliva virgen en España. Un analisis de su eficiencia tecnica. ITEA 2:208-222 (in Spanish) 